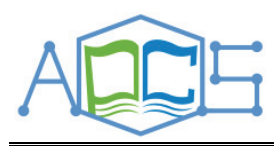

[Highlight]

\title{
功能化离子液体吸收活化二氧化碳制取甲酸
}

韩布兴

中国科学院化学研究所, 胶体、界面与化学热力学实验室, 北京 100190

\section{Absorption and Activation of Carbon Dioxide by Functionalized Ionic Liquid for the Preparation of Formic Acid}

\section{HAN Buxing}

CAS Key Laboratory of Colloid and Interface and Thermodynamics, Institute of Chemistry, Chinese Academy of Sciences, Beijing 100190, P. R. China.

Email: hanbx@iccas.ac.cn

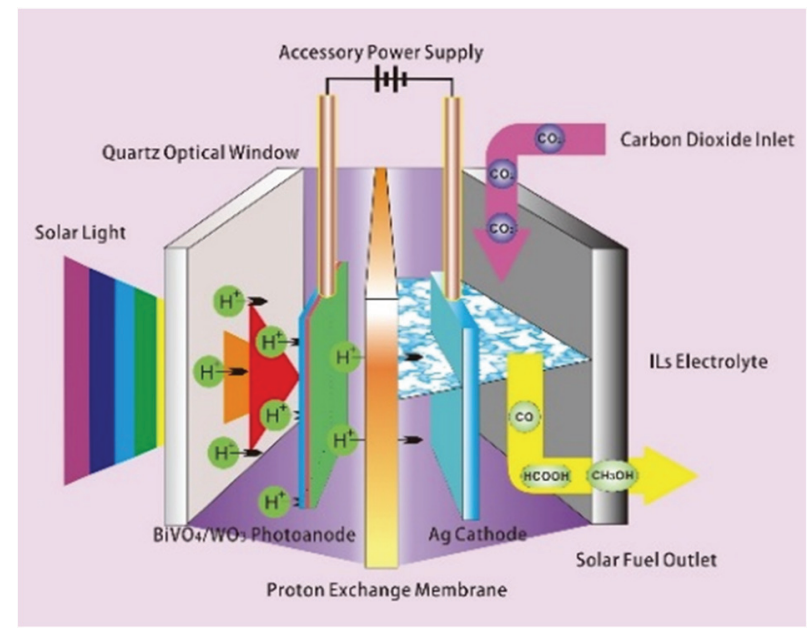

二氧化碳是主要的温室气体, 同时又是重要 的碳资源。因此, 二氧化碳的捕集和利用 $\left(\mathrm{CO}_{2}\right.$ capture and utilization, CCU) 在解决由于化石燃料 的大量使用所导致的环境和能源问题等方面具有 重要的意义 ${ }^{1-3}$ 。与传统的二氧化碳捕获和存储 $\left(\mathrm{CO}_{2}\right.$ capture and storage, $\left.\mathrm{CCS}\right)$ 技术相比 ${ }^{4}, \mathrm{CCU}$ 强调将捕获的 $\mathrm{CO}_{2}$ 作为一种碳资源转化为化学品 和化学燃料, 最终达到碳循环的目的。然而, $\mathrm{CCU}$ 技术面临的主要挑战在于: 在 $\mathrm{CO}_{2}$ 捕集方面, 需 要寻求环境友好、捕集容量高、选择性好, 且易于 脱附再生的捕获剂 ${ }^{5,6}$; 而在 $\mathrm{CO}_{2}$ 的利用方面, 开 发 $\mathrm{CO}_{2}$ 高效转化的催化方法则是需要解决的另一 个关键问题 ${ }^{7-9}$ 。

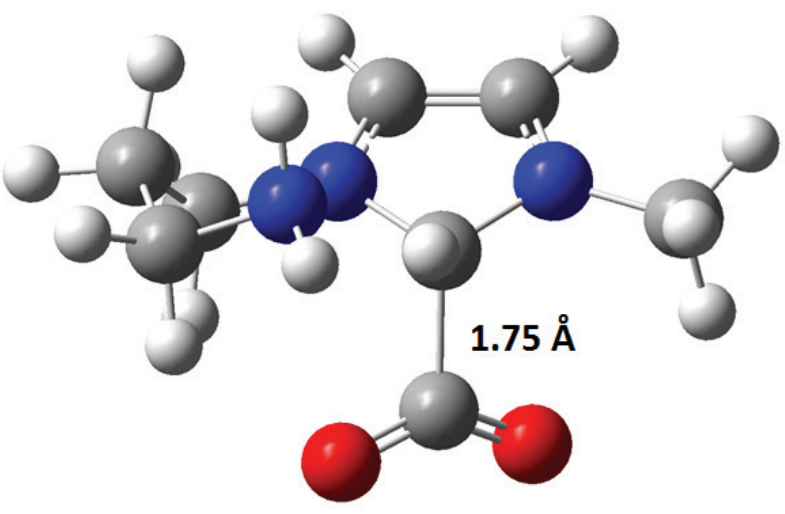

最近, 河南师范大学化学化工学院绿色化学 介质与反应教育部重点实验室王键吉教授课题组 以功能化离子液体作为吸收剂和电解质, 通过光 电化学还原方法成功地将 $\mathrm{CO}_{2}$ 高效转化为甲酸。研究 结果表明, 在以功能化离子液体澳化 1-胺丙基-3-甲基 咪唑 (1-aminopropyl-3-methylimidazolium bromide, $\left.\left[\mathrm{NH}_{2} \mathrm{C}_{3} \mathrm{MIm}\right][\mathrm{Br}]\right)$ 水溶液 $\left(0.454 \mathrm{~mol} \cdot \mathrm{L}^{-1}\right)$ 为 $\mathrm{CO}_{2}$ 的 吸收剂和电解质, $\mathrm{WO}_{3} / \mathrm{BiVO}_{4}$ 复合薄膜为光阳极, 银片为电阴极的光电反应体系中, $\mathrm{CO}_{2}$ 的溶解度达 到 $0.211 \mathrm{~mol} \cdot \mathrm{L}^{-1}$, 且在外加电压为 $1.7 \mathrm{~V}$ 时产物甲 酸的法拉第效率达到 $94.1 \%$, 电能到化学能的转化 效率达到 86.2\%。电化学循环伏安实验结果表明, 与传统的 $\mathrm{KHCO}_{3}$ 和非功能化的溴化 1-丁基-3-甲 
基咪唑 (1-butyl-3-methylimidazolium bromide, $\left.\left[\mathrm{C}_{4} \mathrm{MIm}\right][\mathrm{Br}]\right)$ 离子液体电解质相比, $\mathrm{CO}_{2}$ 在 $\left[\mathrm{NH}_{2} \mathrm{C}_{3} \mathrm{MIm}\right][\mathrm{Br}]$ 水溶液中的起始还原电位明显正 移, 说明了功能化离子液体 $\left[\mathrm{NH}_{2} \mathrm{C}_{3} \mathrm{MIm}\right][\mathrm{Br}]$ 具有 较强的活化 $\mathrm{CO}_{2}$ 的能力。密度泛函理论计算结果 进一步表明, 电极表面吸附的离子液体阳离子 $\left[\mathrm{NH}_{2} \mathrm{C}_{3} \mathrm{MIm}\right]_{(\mathrm{ad})}^{+}$的 2 位 $\mathrm{C}$ 原子和 $\mathrm{CO}_{2}$ 之间较强的 相互作用对活化 $\mathrm{CO}_{2}$ 起到了重要的作用。不同外 加电压条件下的电能-化学能、光能-化学能转化效 率的计算结果表明, 在 $1.1 \mathrm{~V}$ 的外加电压条件下, 光能-化学能的转化效率可以达到 $0.11 \%$ 。此结果 也进一步说明, 将太阳能引入到 $\mathrm{CO}_{2}$ 光电转化体 系中, 可以有效地补偿 $\mathrm{CO}_{2}$ 还原反应的过电位和 体系阻抗造成的电能消耗。

该研究工作近期已在 Angewandte Chemie

International Edition 上在线发表 10 。该研究集绿 色溶剂离子液体对 $\mathrm{CO}_{2}$ 的捕集和转化于一体, 利 用温室气体 $\mathrm{CO}_{2}$ 的光电化学还原制取高附加值的 化学品, 为实现 $\mathrm{CO}_{2}$ 的有效转化和利用提供了一 种新的思路。

\section{References}

(1) Wang, W.; Himeda, Y.; Muckerman, J. T.; Manbeck, G. F.; Fujita, E.
Chem. Rev. 2015, 115, 12936. doi: 10.1021/acs.chemrev.5b00197

(2) Markewitz, P.; Kuckshinrichs, W.; Leitner, W.; Linssen, J.; Zapp, P.; Bongartz, R.; Schreiber, A.; Muller, T. E. Energy Environ. Sci. 2012, 5, 7281. doi: 10.1039/C2EE03403D

(3) Sakakura, T.; Choi, J. C.; Yasuda, H. Chem. Rev. 2007, 107, 2365. doi: $10.1021 / \mathrm{cr} 068357 \mathrm{u}$

(4) Boot-Handford, M. E.; Abanades, J. C.; Anthony, E. J.; Blunt, M. J.; Brandani, S.; Mac Dowell, N.; Fernandez, J. R.; Ferrari, M. C.; Gross, R.; Hallett, J. P.; et al. Energy Environ. Sci. 2014, 7, 130. doi: 10.1039/c3ee42350f

(5) Zeng, S.; Zhang, X.; Bai, L.; Zhang, X.; Wang, H.; Wang, J.; Bao, D.; Li, M.; Liu, X.; Zhang, S. Chem. Rev. 2017, 117, 9625. doi: 10.1021/acs.chemrev.7b00072

(6) Koronaki, I. P.; Prentza, L.; Papaefthimiou, V. Renew. Sustainable Energy Rev. 2015, 50, 547. doi: 10.1016/j.rser.2015.04.124

(7) Chu, S.; Cui, Y.; Liu, N. Nat. Mater. 2017, 16, 16. doi: $10.1038 / \mathrm{nmat} 4834$

(8) Lewis, N. S. Science 2016, 351, 353, doi: 10.1126/science.aad5117

(9) Hernandez, S.; Farkhondehfal, M. A.; Sastre, F.; Makkee, M.; Saracco, G.; Russo, N. Green Chem. 2017, 19, 2326. doi: $10.1039 / \mathrm{c} 7 \mathrm{gc} 00398 \mathrm{f}$

(10) Lu, W.; Jia B.; Cui, B.; Zhang, Y.; Yao, K.; Zhao, Y.; Wang, J. Angew. Chem. Int. Ed. 2017, doi: 10.1002/anie.201703977 